\title{
Copy Move Forgery Detection Using Hybrid Algorithm
}

\author{
Dr. Tekuru Chandra Sekhar Rao ${ }^{1}$, Dr. Sateesh Kumar Kanagala ${ }^{2 *}$, \\ Kancherla Vijaya Vardhan ${ }^{3}$ \\ ${ }^{1}$ Professor, Sri Venkateswara Engineering College, Tirupati, India, chandrasekhar.t@ svcolleges.edu.in ${ }^{1}$ \\ ${ }^{2}$ Assistant Professor, Sreenidhi Institute of Science and Technology, Hyderabad, India, \\ sateeshk@sreenidhi.edu.in ${ }^{2}$ \\ ${ }^{3}$ Assistant Professor, Vignan's LARA Institute of Technology and Science, Guntur, India, \\ kvvardhan405@gmail.com ${ }^{3}$
}

\begin{abstract}
Image forgery detection in terms of copy-move detection is an emerging area for researchers in recent times. This paper proposes the combined methodology of Saliency detection and Local binary pattern-based forgery detection for real time images. Saliency detection is used to identify the forged portion on a preliminary step. It locks the region of the tampered portion with surrounding (global) area. Then, the exact pixels/region is identified or captured by Local Binary Pattern features for the tampered portions. This combined approach maintains the advantages of both saliency map and Local Binary Pattern particularly in scaling/rotation with fast detection rate than the existing methods. In addition to the existing outcome of forgery detection, the proposed method accurately identifies the forged region with depth map information. The severity of the tampered portion is examined with reference to the bits/pixel. The novelty in the proposed method lays the way of detecting the tampered portion in the real-time digital image. The state of art comparison is also proved that the proposed method is far better than existing methods.
\end{abstract}

Key words : Copy-move forgery detection, Image forgery detection, Local binary pattern method, Saliency depth map.

\section{INTRODUCTION}

From last four decades, digital image processing techniques are predominantly working to reform the trust towards the digital images. Undoubtedly, the integrity of digital images is degraded in recent times. Forgery is one of the main reasons for this degradation. Digital watermarking has contributed to address this issue but not up to the mark due to practical difficulties since digital watermarking can be done only at the time of the capturing/recording of an image [1]. This drawback is predominantly dealt by passive techniques where some clues available about the tampering not visible like watermark [2]. Image forgery classified into three categories, they are Image retouching, image splicing and copy-move [3]. This paper addresses the copy move type of image forgery in which some part of the image is copied and moved to other portion of the image. In general, this part is cropped, rotated, and scaled version of the original [4]. The forgery detection methods are divided into two types i.e., Active (non-blind) and Passive (blind). Active methods are watermark based, they have hint about the authenticity or originality. For passive methods there is no information about the genuinety [5],[6]. The proposed forgery detection method belongs to passive type. In this proposed forgery detection method, Saliency based depth map algorithm is applied to identify the exact region of forgery. Then LBP based front layer pixels are identified block wise in each RGB plane of forged portions. This forgery detection method can be applied in medical, forensics, and media to authenticate the integrity of the original image.

This paper is organized in such a way that section 2 briefly presents the literature or related work; section 3 discusses the proposed methodology followed by results and discussion represented in section 4. Finally, the conclusion is in section 5.

\section{RELATED WORK}

Several researchers have effortlessly worked on this problem and achieved remarkable milestones too. Some of the literature is presented briefly in this section. Sun et al. [7] proposed wavelet based statistical method for identifying similar and tampered portions using Gaussian model distribution. Wavelets were also effectively utilized to extract the patterns of gray scale image to detect the tampered portion. DWT and SVD combined blind forgery detection was successfully implemented by $\mathrm{Li}$ et al [8] specifically for highly compressed images. Bravo [9] proposed forgery detection even if image was geometrically distorted such as reflection, scaling, rotation etc. Christlein et al. [10] has set a benchmark dataset for copy detection using Kd Trees based segmentation. Standard LBP and Multi-resolution LBP for colour images 
[11] were also implemented with SIFT to cluster the neighborhood pixels of minimum deviation [12].

The advantage of this method was best for detecting copy area even close to the original pixels. The main drawback was contrast limitation [13] but obtained good performance for JPEG images. Most of these existing methods have considered block level rather than colour distribution at the pixel level for finding copy move detection. In this proposed method colour information was also considered with saliency map for exact and high accurate identification of the tempered region. Davarzani et al. [14] proposed the LBP and neighborhood clustering forgery detection method, where clustering is not the approach for high contrast image, as high chance of false clustering can occur. In this histogram levels are compared and grouped the minimum distance blocks.

\section{PROPOSED METHOD}

In the proposed method, the forgery detection is done using two steps. For tracking of the global forgery region saliency depth map is used and local region is identified with LBP technique based on features. Saliency detection is the best tool to identify the copy paste forgery detection even at minute regions also. The steps of the proposed hybrid forgery copy move detection is presented in figure 1.

\subsection{Saliency Depth Map}

Saliency map is one the fastest techniques used in computer vision applications for image registration and image matching etc. Here, space based visual saliency map [16] is used to identify the copy move forged portions with high dynamic range. The general representation of the saliency map is represented in equation 1.

$$
S_{r c}\left(r_{k}\right)=\sum_{r k \neq r i} \exp \left(-\frac{D_{s}\left(r_{k}, r_{i}\right)}{\delta^{2}}\right) w\left(r_{i}\right) D_{c}\left(r_{k}, r_{i}\right)
$$

where $r_{k}$ and $r_{i}$ represents the segmented regions. $W\left(r_{i}\right)$ is weight region specified by $r_{i} D_{c}\left(r_{k}, r_{i}\right)$ is colour information between regions $r_{k}$ and $r_{i}$ respectively. The spatial weights depend on the image attributes. $\delta$ is variance parameter $(0,1)$.

This saliency map finds the forgery detection at the basic level with the help of depth map. The forged portions always differ in depth map from its neighbors which cannot be observed in the visual RGB plane. This can be visually represented in figures of results section.

\subsection{Local Binary Pattern (LBP)}

LBP technique is a texture operator on pixel level to assign labels based on their gray levels by considering its neighbors. The radius defines the number of neighbors it can consider assigning a label. The output of LBP operator is binary only. In the proposed methodology circular sampling was done.

$\operatorname{LBP}\left(r_{k f}, r_{i f}\right)=\sum_{p=1}^{P} S\left(g_{p}, g_{c}\right) 2^{p} s(x)=[1, x \geq 0 ; 0,<0]$

where, $r_{h f}$ and $r_{c f}$ are features of regions divided by saliency map techniques.

$g_{p}$ is the gray value of the subjected pixel. $g_{s}$ is the gray level of the center pixel.

$\mathrm{P}$ is the sampling points (defined by radius) within the region.

This LBP helps to discriminate the portions of forgery in gray level plane. In fact, LBP is time taking process as it applies on whole image. But, in this proposed hybrid method of forgery detection; the area is limited by saliency map which saves the computation time.

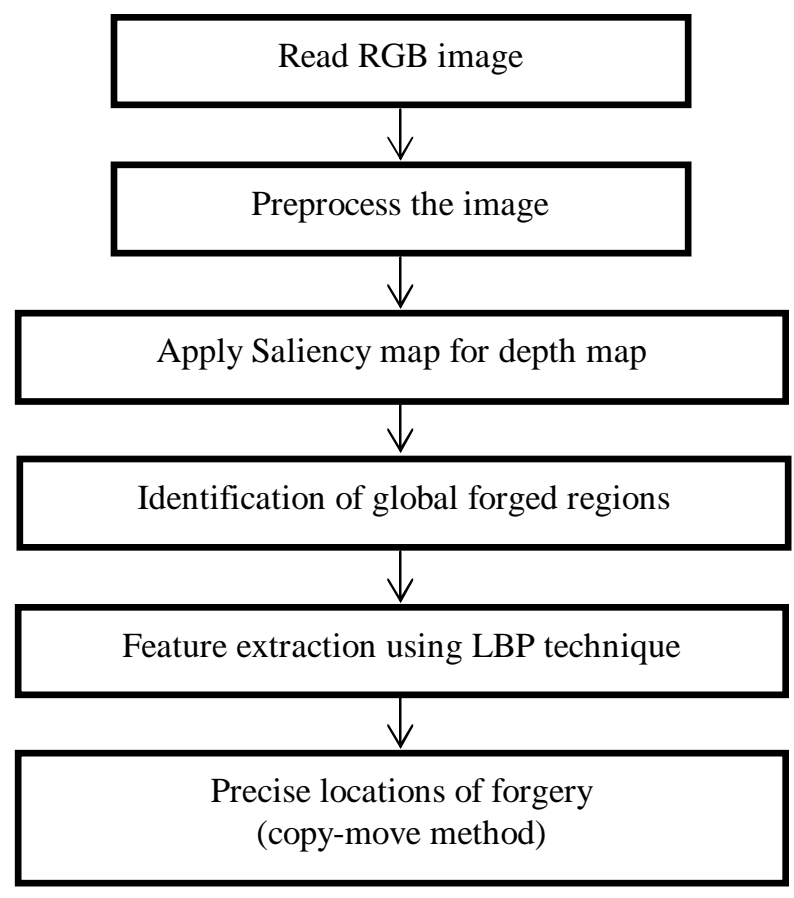

Figure 1: Methodology of the proposed forgery detection

\section{RESULTS AND DISCUSSION}

This section deals with the results of the proposed method and comparison of the performance metrics with the existing techniques. The detection of copy paste region is only with the help of reference image only. So, it is a blind process to identify forged portion. This proposed method obtained the better performance than solely LBP or Saliency based copy paste detection. Computation time is also saved significantly 
by this hybrid approach of copy move algorithm. The images are collected from standard database. From this database more than 150 real-time images are tested by applying the proposed algorithm and tabulated the results. Different operations like JPEG compression, rotation and flipping are done on these images.

Along with the state of art performance metrics [17] computation time is compared with the existing forgery detection techniques for different set of images. Preferred performance metrics are represented in equations (3) and (4) respectively.

Pr ecision $=\frac{\text { TruePositi ve }}{\text { TruePositi ve }+ \text { FalsePosit ive }}$

$\operatorname{Re}$ call $=\frac{\text { TruePositi ve }}{\text { TruePositi ve }+ \text { FalseNegat ive }}$

The terms True Positive (TP), True Negative (TN), False Positive (FP) and False Negative (FN) are represented in table 1. The horizontal bottom line represents the input regions either forged or not forged portions. The vertical column represents the detected portions of possible output form the proposed algorithm. The combination of actual and estimated frames cases of TN.TP, FP and FN. For a better algorithm, the TP is as much as high (at least, >95\%) and FP as much as minimum (at most. < 10\%). These values depend on the number of images considered from the database. Large database is preferred over numerical values to overcome ambiguity between two classes i.e., forged and not forged.
Table 2 represents the comparison of the performance metrics under different conditions like compression and rotation. It is more efficient than the existing forgery detection techniques like pure saliency and pure LBP. In addition to the identification of the forged portion depth of pixels is also find by the saliency map. The scope of LBP match with the neighboring regions is also expressed in number between 0 and 1 . If it is 1 , not forged and 0 means forged portion detected.

Table 1: Format of Confusion Matrix

\begin{tabular}{|c|c|c|}
\hline $\begin{array}{l}\text { D0 } \\
\text { o0 } \\
0 \\
0 \\
0 \\
Z\end{array}$ & False Positive (FP) & True Negative (TN) \\
\hline \multirow{2}{*}{ 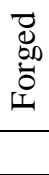 } & True Positive (TP) & False Negative $(\mathrm{FN})$ \\
\hline & Forged & Not forged \\
\hline
\end{tabular}

This is an advantage than the existing forgery detection techniques. Figure 2 shows the original and forged images. Figure 3 represents the forged portion in which trees are copied and moved to portion where building exists. This portion is highlighted by box representation. In a similar way figure 5 and figure 6 represents the forgery(copy-move) in which ships are copied and moved to another place in the image.

Table 2: Comparison of the Performance Metrics

\begin{tabular}{|c|l|c|c|c|c|c|c|}
\hline \multicolumn{2}{|c}{} & \multicolumn{2}{c|}{ Image 1 } & \multicolumn{2}{c|}{ Image 2 } & \multicolumn{2}{c|}{ Image 3 } \\
\hline \multirow{2}{*}{ Effect } & Methods & Precision & Recall & Precision & Recall & Precision & Recall \\
\hline \multirow{3}{*}{$\begin{array}{c}\text { Without } \\
\text { any effect }\end{array}$} & Proposed & 0.89 & 0.7 & 0.97 & 0.84 & 0.99 & 0.92 \\
\cline { 2 - 8 } & LBP [15] & 0.72 & 0.87 & 0.74 & 0.87 & 0.82 & 0.87 \\
\cline { 2 - 9 } & Saliency [17] & 0.79 & 0.8 & 0.8 & 0.72 & 0.93 & 0.91 \\
\hline \multirow{3}{*}{$\begin{array}{c}\text { JPEG } \\
\text { Compression }\end{array}$} & Proposed & 0.78 & 0.66 & 0.83 & 0.86 & 0.9 & 0.77 \\
\cline { 2 - 8 } & LBP [15] & 0.69 & 0.62 & 0.79 & 0.75 & 0.76 & 0.84 \\
\cline { 2 - 8 } & Saliency [17] & 0.73 & 0.7 & 0.82 & 0.85 & 0.78 & 0.63 \\
\hline \multirow{4}{*}{ Rotation } & Proposed & 0.85 & 0.65 & 0.88 & 0.84 & 0.94 & 0.79 \\
\cline { 2 - 8 } & LBP [15] & 0.72 & 0.59 & 0.64 & 0.77 & 0.75 & 0.86 \\
\cline { 2 - 8 } & Saliency [17] & 0.8 & 0.66 & 0.81 & 0.79 & 0.89 & 0.9 \\
\hline
\end{tabular}


Tekuru Chandra Sekhar Rao et al., International Journal of Advanced Trends in Computer Science and Engineering, 9(4), July - August 2020, 5071 - 5076

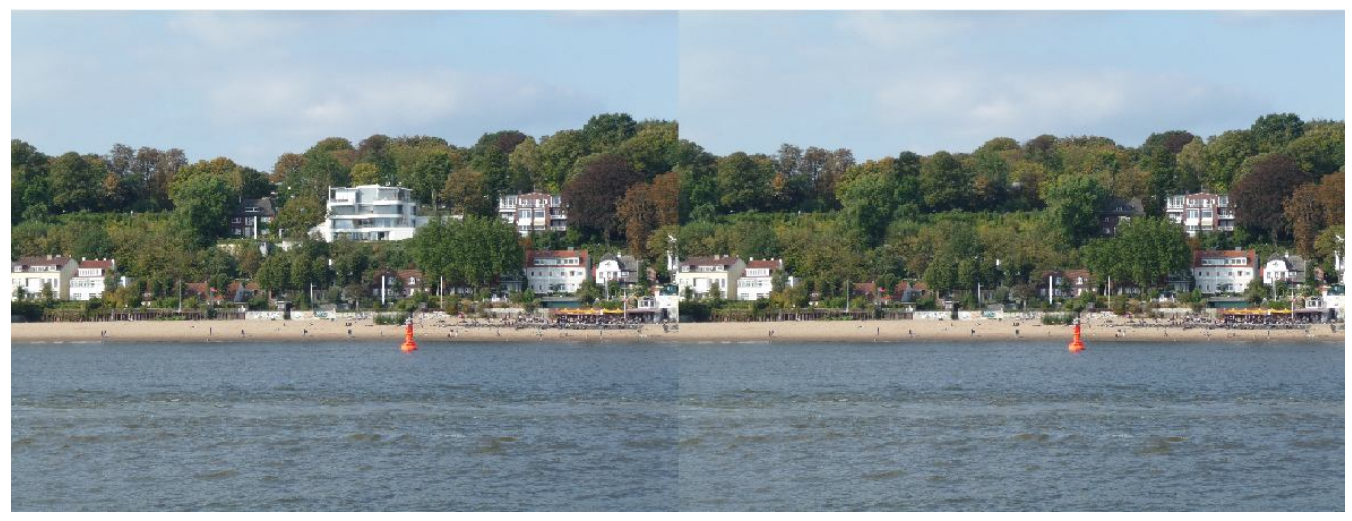

Figure 2: Original Image and Forged Image (from left to right)

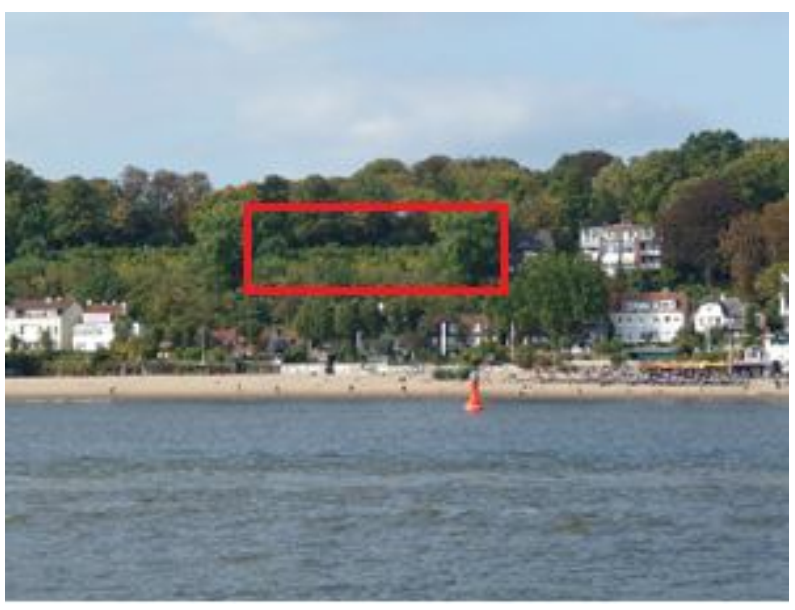

Figure 3: Forged portion of figure 2.

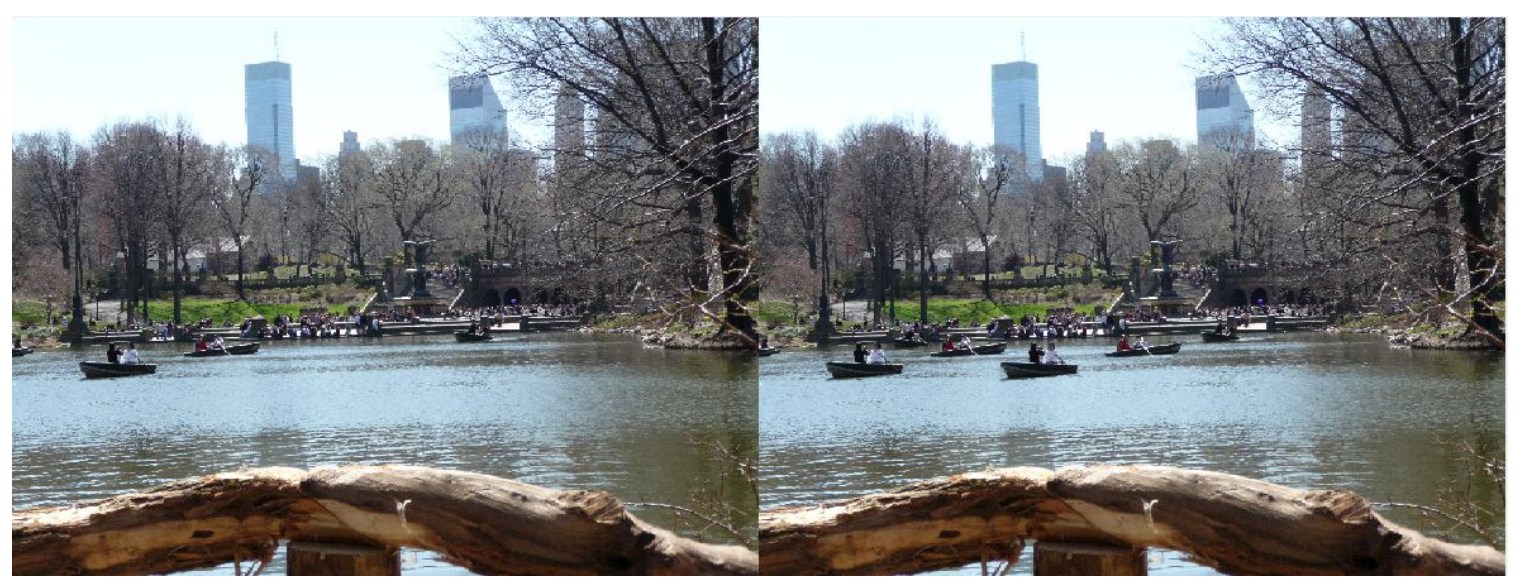

Figure 4: Original image and forged image (from left to right) 


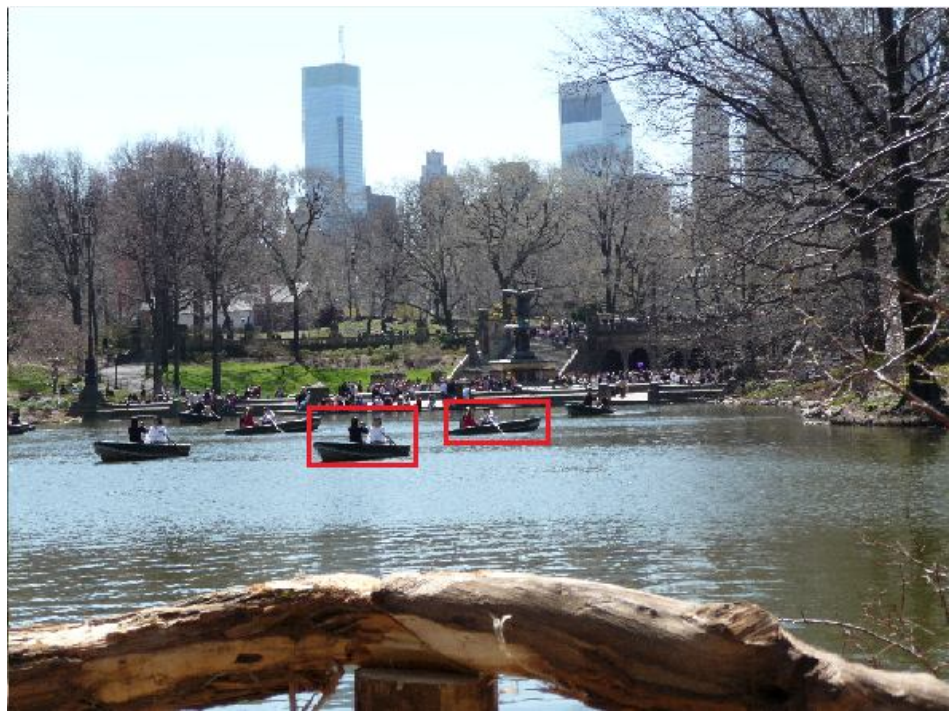

Figure 5: Forged portion of figure 4.

\section{CONCLUSION}

In this paper, the proposed methodology is better than existing techniques. It is achieved due to block wise processing of the colour image in different colour planes instead of RGB alone. The proposed methodology significantly dominates the existing methods even in compression, rotating and flipping scenarios also. By applying saliency detection technique, the primary level of forgery was found and fine-tuned with the help of LBP. Instead of applying LBP on whole image, it is operated on the preliminary portions of the forgery. This is the reason where it is better in the computation time also.

\section{REFERENCES}

1. Chandramouli, Rajarathnam, Memon, Nasir and Rabbani, Majid. Digital Watermarking, Springer, 2012.

2. Amir Hesam Yaribakht, Mohd Shahidan Abdullah, and Alireza Ghobadi. Novel Color Image Watermarking Method based on Digital Wavelet Transform and Hungarian Algorithms, International Journal of Advanced Trends in Computer Science and Engineering, Volume 8, No.2, 2020. https://doi.org/10.30534/ijatcse/2019/09822019

3. M. AlSawadi, G. Muhammad, M. Hussain and G. Bebis. Copy-Move Image Forgery Detection Using Local Binary Pattern and Neighborhood Clustering, Proc. European Modelling Symposium, Manchester, 2013, pp. 249-254.

4. A. Thakur and N. Jindal. Machine Learning Based Saliency Algorithm For Image Forgery Classification And Localization, Proc. 1st International Conference on Secure Cyber Computing and Communication (ICSCCC), Jalandhar, India, 2018, pp. 451-456.

5. Kaushik H. Raviya, Dr. Diwedi Ved Vyas, and Dr. Asish M. Kothari. SVD Based Performance Improvement in Hiding a Message Behind an Image, International Journal of Advanced Trends in Computer Science and Engineering, Vol. 8,2019

https://doi.org/10.30534/ijatcse/2019/12822019

6. F. Peng, Y. Y. Nie and M. Long. A complete passive blind image copy-move forensics scheme based on compound statistics features, Forensic Science International, Vol.210, pp. e21-e25,2002.

7. S.J. Sun, Q. Wu and G.H. Li. Detection of image compositing based on a statistical model for natural images, Acta Automatica Sinica, Vol. 35, No. 12, pp. 1564-1568, 2009

8. G. Li, Q. Wu, D. Tu and S .Sun. A sorted neighborhood approach for detecting duplicated regions in image forgeries based on DWT and SVD, Proc. International Conference on Multimedia and Expo (ICME),2007, pp. $1750-1753$.

9. S. Bravo-Solorio and A. K. Nandi. Passive forensic method for detecting duplicated regions affected by reflection, rotation and scaling, Proc. 17th European Signal Processing Conference (EUSIPCO),2009, pp.824-828.

10. V. Christlein, C. Riess and E. Angelopoulou. A Study on Features for the Detection of Copy-Move Forgeries, Proc. Information Security Solution Europe (ISSE),2010 pp. 105-116.

11. Y. Cao, T. Gao, L. Fan, Q. Yang, A robust detection algorithm for copy-move forgery in digital images, Forensic Science International, Vol. 214, pp. 33-43,2012.

https://doi.org/10.1016/j.forsciint.2011.07.015 
12. Xi T, Zhao W, Wang H, Lin W. Salient object detection with spatiotemporal background priors for video, IEEE Trans Image Process, Vol 26(7),pp.3425-3436, 2012

13. Ullah, I., Jian, M., Hussain, S. et al. A brief survey of visual saliency detection, Multimedia Tools Application, Vol.10, 2020.

14. R. Davarzani, K. Yaghmaie, S. Mozaffari, and M. Tapak. Copy-move forgery detection using multiresolution local binary patterns, Forensics Science International, vol. 231, pp. 61-72,2013.

15. Mahale, Vivek \& Ali, Mouad \& Yannawar, Pravin \& Gaikwad, Ashok. Image Inconsistency Detection Using Local Binary Pattern. Procedia Computer Science. 2018,pp. 501-508.

https://doi.org/10.1016/j.procs.2017.09.097

16. Zou, Xiaochun \& Zhao, Xinbo \& Yang, Yongjia \& Li, Na. Learning-Based Visual Saliency Model for Detecting Diabetic Macular Edema in Retinal Image, Computational Intelligence and Neuroscience. Vol. 21, 2016.

17. S. M. Muddamsetty, D. Sidibé, A. Trémeau and F. Mériaudeau. Spatio-temporal Saliency Detection in Dynamic Scenes Using Local Binary Patterns, Proc. $22^{\text {nd }}$ International Conference on Pattern Recognition, Stockholm, 2014, pp. 2353-2358.

https://doi.org/10.1109/ICPR.2014.408 